\title{
Magnetogenesis and the dynamics of internal dimensions
}

\author{
Massimo Giovannini* \\ Institute of Theoretical Physics, University of Lausanne \\ BSP-1015 Dorigny, Lausanne, Switzerland
}

\begin{abstract}
The dynamical evolution of internal space-like dimensions breaks the invariance of the Maxwell's equations under Weyl rescaling of the (conformally flat) four-dimensional metric. Depending upon the number and upon the dynamics of internal dimensions large scale magnetic fields can be created. The requirements coming from magnetogenesis together with the other cosmological constraints are examined under the assumption that the internal dimensions either grow or shrink (in conformal time) prior to a radiation dominated epoch. If the internal dimensions are growing the magnitude of the generated magnetic fields can seed the galactic dynamo mechanism.
\end{abstract}

Preprint Number: UNIL-IPT-00-16, June 2000

*Electronic address: Massimo.Giovannini@ipt.unil.ch 


\section{INTRODUCTION}

The past dynamical history of our Universe is not completely known and the theoretical understanding is often guided by consistency rather than by observational evidence. The remarkable similarity of the abundances of light elements in different galaxies leads to postulate that the Universe had to be dominated by radiation at the moment when the light elements were formed, namely for temperatures of approximately $0.1 \mathrm{MeV}$ [1]. Prior to the moment of nucleosynthesis even indirect informations concerning the thermodynamical state of our Universe are lacking even if our knowledge of particle physics could give us important hints concerning the dynamics of the electroweak phase transition [2].

The success of big-bang nucleosynthesis (BBN) sets limits on alternative cosmological scenarios. Departures from homogeneity [3] and isotropy [4] of the background geometry can be successfully constrained. Bounds on the presence of matter-antimatter domains of various sizes can be derived [5,6]. BBN can also set limits on the dynamical evolution of internal dimensions [7,8]. Internal dimensions are an essential ingredient of theories attempting the unification of gravitational and gauge interactions in a higher dimensional background like Kaluza-Klein theories [9] and superstring theories [10].

Defining, respectively, $b_{B B N}$ and $b_{0}$ as the size of the internal dimensions at the BBN time and at the present epoch, the maximal variation allowed to the internal scale factor from the BBN time can be expressed as $b_{B B N} / b_{0} \sim 1+\epsilon$ where $|\epsilon|<10^{-2}$ [7, 8]. The bounds on the variation of the internal dimensions during the matter dominated epoch are even stronger. Denoting with an over-dot the derivation with respect to the cosmic time coordinate, we

have that $|\dot{b} / b|<10^{-9} H_{0}$ where $H_{0}$ is the present value of the Hubble parameter [7]. The fact that the time evolution of internal dimensions is so tightly constrained for temperatures lower of $1 \mathrm{MeV}$ does not forbid that they could have been dynamical prior to that epoch.

An apparently unrelated observational evidence characterizing the present Universe is the existence of large scale magnetic fields [11. Faraday rotation measurements, Zeeman splitting estimates (when available) and synchrotron emission patterns indicate that distant 
galaxies seem to be endowed with a magnetic field of roughly the same strength of the one of the Milky Way [13,14] which is of the order of $10^{-6}$ G. The similarity of the magnetic field strength in different galaxies led to the feeling that the origin of these large scale fields should somehow be connected with the cosmological consequences of the interplay between gravitational and gauge interactions [12]. Observations of magnetic fields at even larger scales (i.e cluster, inter-cluster) are still under debate mainly because of the rather problematic estimates of the electron density in the inter-galactic medium [14. The existence of strong magnetic fields with coherence scale larger than the galactic one can be of crucial importance for the propagation of high-energy cosmic rays.

The measured large scale (galactic) magnetic fields are assumed to be the result of the exponential amplification (due to galactic rotation) of some primeval seed fields [12,15] whose typical value lies around $10^{-16}-10^{-25} \mathrm{G}$ at the decoupling epoch. Several mechanisms have been invoked in order to explain the origin of these seeds [16 18]. Large scale magnetic fields can also have relevant physical implications in other (related) areas of cosmology and especially in connection with polarization (and distortion) of the Cosmic Microwave Background (CMB) [19].

In the present paper it is argued that there may be a relation between the existence of large scale magnetic fields and the possible occurrence of a primordial phase of the Universe where the internal dimensions have been dynamical. In short the logic goes as follows. Suppose that prior to BBN internal dimensions were evolving in time and assume, for sake of simplicity, that after BBN the internal dimensions have been frozen to their present (constant) value. The evolution in time of a classical cosmological background can amplify a given distribution of (initially small) inhomogeneities of the metric and of (non conformally coupled) matter fields [20,21]. If the background geometry is isotropic and conformally flat Abelian gauge fields cannot be amplified since their equations of motion are invariant under a (Weyl) rescaling of the metric tensor. However, if the internal dimensions change in time the Weyl invariance of Maxwell equations is naturally broken [22] and electromagnetic (vacuum) fluctuations can be amplified. 
The purpose of the present investigation is to clarify if a suitable dynamics of the internal dimensions can produce sizable seed fields which could turn on the galactic dynamo mechanism and have other indirect effects during the evolution of the Universe. The plan of the paper is then the following. In Section II we will discuss our basic equations. In Section III we will perform a model independent analysis of the amplification of magnetic fields from monotonically evolving internal dimensions. In Section IV some specific models of internal evolution will be studied. Section V contains the concluding remarks

\section{BASIC EQUATIONS}

Consider a homogeneous and anisotropic manifold whose line element can be written as

$$
\begin{array}{r}
d s^{2}=G_{\mu \nu} d x^{\mu} d x^{\nu}=a^{2}(\eta)\left[d \eta^{2}-\gamma_{i j} d x^{i} d x^{j}\right]-b^{2}(\eta) \gamma_{a b} d y^{a} d y^{b} \\
\mu, \nu=0, \ldots, D-1=d+n, \quad i, j=1, \ldots, d, \quad a, b=d+1, \ldots, d+n .
\end{array}
$$

$\left[\eta\right.$ is the conformal time coordinate related, as usual to the cosmic time $t=\int a(\eta) d \eta ; \gamma_{i j}(x)$, $\gamma_{a b}(y)$ are the metric tensors of two maximally symmetric Euclidean manifolds parameterized, respectively, by the "internal" and the "external" coordinates $\left\{x^{i}\right\}$ and $\left.\left\{y^{a}\right\}\right]$. The metric of Eq. (2.1) describes the situation in which the $d$ external dimensions (evolving with scale factor $a(\eta))$ and the $n$ internal ones (evolving with scale factor $b(\eta)$ ) are dynamically decoupled from each other [23]. The results of the present investigation, however, can be easily generalized to the case of $n$ different scale factors in the internal manifold.

Consider now a pure electromagnetic fluctuation decoupled from the sources, representing an electromagnetic wave propagating in the $d$-dimensional external space such that $A_{\mu} \equiv$ $A_{\mu}(\vec{x}, \eta), A_{a}=0$. In the metric given in Eq. (2.1) the evolution equation of the gauge field fluctuations can be written as

$$
\frac{1}{\sqrt{-G}} \partial_{\mu}\left(\sqrt{-G} G^{\alpha \mu} G^{\beta \nu} F_{\alpha \beta}\right)=0,
$$

where $F_{\alpha \beta}=\nabla_{[\alpha} A_{\beta]}$ is the gauge field strength and $G$ is the determinant of the $D$ dimensional metric. Notice that if $n=0$ the space-time is isotropic and, therefore, the Maxwell's 
equations can be reduced (by trivial rescaling) to the flat space equations. If $n \neq 0$ we have that the evolution equation of the electromagnetic fluctuations propagating in the external $d$-dimensional manifold will receive a contribution from the internal dimensions which cannot be rescaled away [22] In. In the radiation gauge $\left(A_{0}=0\right.$ and $\left.\nabla_{i} A^{i}=0\right)$ f the evolution the vector potentials can be written as

$$
A_{i}^{\prime \prime}+n \mathcal{F} A_{i}^{\prime}-\vec{\nabla}^{2} A_{i}=0, \quad \mathcal{F}=\frac{b^{\prime}}{b}
$$

The vector potentials $A_{i}$ are already rescaled with respect to the (conformally flat) $d+1$ dimensional metric. In terms of the canonical normal modes of oscillations $\mathcal{A}_{i}=b^{n / 2} A_{i}$ the previous equation can be written in a simpler form, namely

$$
\mathcal{A}_{i}^{\prime \prime}-V(\eta) \mathcal{A}_{i}-\vec{\nabla}^{2} \mathcal{A}_{i}=0, \quad V(\eta)=\frac{n^{2}}{4} \mathcal{F}^{2}+\frac{n}{2} \mathcal{F}^{\prime}
$$

In order to estimate the amplification of the gauge fields induced by the evolution of the internal geometry we shall consider the background metric of Eq. (2.1) in the case of maximally symmetric subspaces $\gamma_{i j}=\delta_{i j}, \gamma_{a b}=\delta_{a b}$.

Suppose now that the background geometry evolves along three different epochs. During the first phase (taking place for $\left.]-\infty,-\eta_{1}\right]$ ) the evolution is truly multidimensional. At $\eta=-\eta_{1}$ the multidimensional dynamics is continuously matched to a radiation dominated phase turning, after decoupling, into a matter dominated regime of expansion. During the radiation and matter dominated stages the internal dimensions are fixed to their (present) constant size in order not to conflict with possible bounds arising both at the BBN time and during the matter-dominated epoch. The evolution of the external dimensions does not

\footnotetext{
${ }^{1}$ Notice that the electromagnetic field couples only to the internal dimensions through the determinant of the $D$-dimensional metric. In string theories, quite generically, the one-form fields are also coupled to the dilaton field. This case has been already analyzed in the context of string inspired cosmological scenarios [17].

${ }^{2}$ For a discussion of gauges in curved spaces see [24]
} 
affect the amplification of the gauge fields as it can be argued from Eq. (2.4) :in the limit $n \rightarrow 0$ (i.e. conformally invariant background) Eq. (2.4) reduces to the flat space equation.

A model of background evolution can be generically written as

$$
\begin{array}{lc}
a(\eta)=a_{1}\left(-\frac{\eta}{\eta_{1}}\right)^{\sigma}, \quad b(\eta)=b_{1}\left(-\frac{\eta}{\eta_{1}}\right)^{\lambda}, & \eta<-\eta_{1}, \\
a(\eta)=a_{1}\left(\frac{\eta+2 \eta_{1}}{\eta_{1}}\right), \quad b(\eta)=b_{1}, & -\eta_{1} \leq \eta \leq \eta_{2}, \\
a(\eta)=a_{1} \frac{\left(\eta+\eta_{2}+4 \eta_{1}\right)^{2}}{4 \eta_{1}\left(\eta_{2}+2 \eta_{1}\right)}, \quad b(\eta)=b_{1}, & \eta>\eta_{2} .
\end{array}
$$

In the parameterization of Eq. (2.5) the internal dimensions grow (in conformal time) for $\lambda<0$ and they shrink for $\lambda>0$ ?. By inserting this background into Eq. (2.4) we obtain that for $\eta<-\eta_{1}$

$$
V(\eta)=\frac{n \lambda}{4 \eta^{2}}(n \lambda-2)
$$

whereas $V(\eta) \rightarrow 0$ for $\eta>-\eta_{1}$. Since $V(\eta)$ goes to zero for $\eta \rightarrow \pm \infty$ we can define, in both limits, a Fourier expansion of $\mathcal{A}_{i}$ in terms of two distinct orthonormal sets of modes. If we promote the classical fields to quantum mechanical operators in the Heisenberg representation we can write, for $\eta \rightarrow-\infty$

$$
\hat{\mathcal{A}}_{i}^{\mathrm{in}}(\vec{x}, \eta)=\int \frac{d^{3} k}{(2 \pi)^{3 / 2}} \sum_{\alpha} e_{i}^{\alpha}(\vec{k})\left[a_{k, \alpha} \phi_{k}(\eta) e^{i \vec{k} \cdot \vec{x}}+a_{-k, \alpha}^{\dagger} \phi_{k}^{*}(\eta) e^{-i \vec{k} \cdot \vec{x}}\right],
$$

where the sum runs over the physical polarizations. For $\eta \rightarrow+\infty \mathcal{A}_{i}$ can be expanded in a second orthonormal set of modes

$$
\hat{\mathcal{A}}_{i}^{\text {out }}(\vec{x}, \eta)=\int \frac{d^{3} k}{(2 \pi)^{3 / 2}} \sum_{\alpha} e_{i}^{\alpha}(\vec{k})\left[\tilde{a}_{k, \alpha} \psi_{k}(\eta) e^{i \vec{k} \cdot \vec{x}}+\tilde{a}_{-k, \alpha}^{\dagger} \psi_{k}^{*}(\eta) e^{-i \vec{k} \cdot \vec{x}}\right] .
$$

\footnotetext{
${ }^{3}$ To assume that the internal dimensions are constant during the radiation and matter dominated epoch is not strictly necessary. If the internal dimensions have a time variation during the radiation phase we must anyway impose the BBN bounds on their variation [7,8]. The tiny variation allowed by BBN implies that $b(\eta)$ must be effectively constant for practical purposes.
} 
Since both sets of modes are complete the old modes can be expressed in terms of the new ones

$$
\phi_{k}(\eta)=c_{+}(k) \psi_{k}(\eta)+c_{-}(k) \psi_{k}^{*}(\eta)
$$

If we insert this last equation back into Eq. (2.7) we get

$$
\tilde{a}_{k}=c_{+}(k) a_{k}+c_{-}(k)^{*} a_{-k}^{\dagger}
$$

The commutation relations within the "in" and "out" sets of orthonormal expansions are preserved if the two complex numbers $c_{+}(k)$ and $c_{-}(k)$ (the so-called Bogoliubov coefficients) are subjected to the constraints $\left|c_{+}(k)\right|^{2}-\left|c_{-}(k)\right|^{2}=1$ so that the (unitary) connection between the two asymptotic vacua is parametrized, overall, by three real numbers. If the continuity of the field operators in $-\eta_{1}$ is imposed $c_{ \pm}(k)$ can be determined. The continuity between the two asymptotic forms of the field operators is ensured provided the old mode functions and their first derivatives are continuously matched to the new ones. The evolution equation satisfied by the mode functions during the multidimensional phase

$$
\frac{d^{2} \phi_{k}}{d \eta^{2}}+\left[k^{2}-V(\eta)\right] \phi_{k}=0
$$

can be exactly solved

$$
\phi_{k}(\eta)=\frac{p}{\sqrt{2 k}} \sqrt{k \eta} H_{\nu}^{(2)}(k \eta), \quad p=\sqrt{\frac{\pi}{2}} e^{i \frac{\pi}{4}(1+2 \nu)}
$$

where $H_{\nu}^{(2)}$ is simply the Hankel function of second kind [25]. For $\eta>-\eta_{1}$ the solution of Eq. (2.4) can be simply written as

$$
\phi_{k}(\eta)=c_{+} \psi_{k}(\eta)+\psi_{k}^{*}(\eta), \quad \psi_{k}(\eta)=\frac{1}{\sqrt{2 k}} e^{-i k\left(\eta+\eta_{1}\right)}
$$

The frequency mixing coefficient $c_{-}(k)$ determines the spectral number of produced particles in a given mode of the field which turns out to be $\left\langle n_{k}\right\rangle \sim\left|c_{-}(k)\right|^{2}$. By continuously matching, in $\eta=-\eta_{1}$, the solutions expressed by Eqs. (2.12) and (2.13) the explicit form of the mixing coefficients can be obtained 


$$
c_{\mp}=\frac{p}{2}\left\{H_{\nu}^{(2)}\left(-k \eta_{1}\right)\left[\sqrt{-k \eta_{1}} \mp \frac{i}{\sqrt{-k \eta_{1}}}\left(\nu+\frac{1}{2}\right)\right] \pm i \sqrt{-k \eta_{1}} H_{\nu+1}^{(2)}\left(-k \eta_{1}\right)\right\} .
$$

The mixing coefficients determined in the approximation of a sudden change of the background geometry (taking place in $-\eta_{1}$ ) lead to an ultraviolet divergence in the number and in the energy density of produced particles [26]. In fact for modes of comoving frequency $k^{2}$ larger than the height of the potential barrier $V(\eta)$ [see Eq. (2.6)] the sudden approximation is not adequate. The mixing coefficients, in the regime $k>\sqrt{V}$, should be computed using a smooth function interpolating between the two regimes. This standard analysis [26,27] leads to a number of particle which is exponentially suppressed as $\exp \left[-q k / k_{1}\right]$ where $k_{1} \sim \eta_{1}^{-1}$ and $q$ is a number of order one. Thus, the frequency mixing of modes which never hit the potential barrier will be approximately neglected [28] since, for these modes, $c_{+}(k) \sim 1$ and $c_{-}(k) \sim 0$. The modes experiencing the amplification are the ones for which $k<\sqrt{V}$. Since the maximal height of the barrier is set by the time scale of the transition (i.e. $V\left(\eta_{1}\right) \sim \eta_{1}^{-2}$ ) the results of Eq. (2.14) can be safely estimated in the small argument limit namely for $\left|k \eta_{1}\right|<1$ of the corresponding Hankel functions.

Using the explicit form of the field operators the two-point correlation function of the magnetic field fluctuations can be computed

$$
\mathcal{G}_{i j}(\vec{r}, \eta)=\left\langle 0_{k}\left|\hat{B}_{i}(\vec{x}, \eta) \hat{B}_{j}(\vec{x}+\vec{r}, \eta)\right| 0_{k}\right\rangle=\int d^{3} k \mathcal{G}_{i j}(k) e^{i \vec{k} \cdot \vec{r}}
$$

with

$$
\mathcal{G}_{i j}(k)=\frac{\mathcal{K}_{i j}}{(2 \pi)^{3}} \mathcal{C}(\nu) k\left|k \eta_{1}\right|^{-1-2 \nu}, \quad 2 \nu=|n \lambda-1|
$$

and where

$$
\begin{aligned}
& \mathcal{K}_{i j}=\sum_{\alpha} e_{i}^{\alpha}(k) e_{j}^{\alpha}(k)=\left(\delta_{i j}-\frac{k_{i} k_{j}}{k^{2}}\right), \\
& \mathcal{C}(\nu)=\frac{2^{2 \nu-3}}{\pi} \Gamma^{2}(\nu)\left(\frac{1}{2}-\nu\right)^{2} .
\end{aligned}
$$

The magnetic energy density can be obtained by tracing over the physical polarizations with the result that 


$$
\rho_{B}(r)=\int \rho_{B}(k) \frac{\sin k r}{k r} \frac{d k}{k}
$$

where,

$$
\rho_{B}(k)=\frac{\mathcal{C}(\nu)}{\pi^{2}} k^{4}\left|k \eta_{1}\right|^{-1-2 \nu}
$$

is the logarithmic energy spectrum of the magnetic fluctuations [16, 17] expressed in terms of the comoving wavenumber $k$ which equals the physical momentum of the wave at $\eta=-\eta_{1}$ [we set $a\left(-\eta_{1}\right) \equiv a_{1}=1$ ]. At later times the momentum is given by $\omega(\eta)=k / a(\eta)$ and the wavelength is $2 \pi a(\eta) / k$.

For $\eta<-\eta_{1}$ Eq. (2.4) is written in vacuum (no conductivity is present). As soon as the Universe gets into the radiation dominated phase the conductivity $\sigma_{c}$ suddenly jumps to a finite value of the order of $T_{1}$, namely the temperature right after $-\eta_{1}$. Thus, the electric fields will be rapidly dissipated whereas the magnetic fields will not be damped, namely

$$
\mathcal{B}_{i}\left(\eta_{1}+\Delta \eta\right) \sim \mathcal{B}_{i}\left(\eta_{1}\right), \quad \mathcal{E}_{i}\left(\eta_{1}+\Delta \eta\right) \sim e^{-\sigma_{c} a_{1} \Delta \eta} \mathcal{E}_{i}\left(\eta_{1}\right)
$$

Indeed, when the conductivity jumps to finite value, the magnetic fields survive whereas the electric fields are dissipated in a time $\Delta \eta$ which is proportional to $T_{1}^{-1}$ [29]. This physical situation is different from the one occurring in a tokamak [30 where the system is non-relativistic since the mass of the charge carriers is normally much larger than the temperature of the plasma.

In the absence of vorticity in the bulk velocity field of the plasma (i.e. if $\vec{\nabla} \times \vec{v}=0$ ), a given mode $\mathcal{B}_{i}(\omega)=B_{i}(\omega) a^{2}(\eta)$ of the magnetic field will approximately evolve as

$$
B_{i}(\omega, \eta) a^{2}(\eta) \sim B_{i}\left(\omega, \eta_{1}\right) a^{2}\left(\eta_{1}\right) e^{-\frac{\omega^{2}}{\sigma} \eta}
$$

where $\sigma=\sigma_{c} a(\eta)$ is the (curved-space) form of the conductivity. Provided that $\omega<\omega_{\sigma} \sim$ $\sqrt{\sigma / \eta}$ the magnetic flux is conserved and the magnetic energy density red-shifts as $[a(\eta)]^{-4}$. Magnetic fields whose typical momentum is much smaller than $\omega_{\sigma}$ ( sometimes called magnetic diffusivity scale) correspond to the large scales relevant for the dynamo action. 
If some primordial vorticity is present (i.e. $\vec{\nabla} \times \vec{v} \neq 0$ ) the evolution of the magnetic fields cannot be disentangled from the evolution of the bulk velocity field [13,,31]. The parity breaking (helical) velocity field could be the result of some dynamically developed turbulence [32], of some specific initial conditions [12], or of the gravitational collapse [13]. Indeed, the galaxy is formed with some typical rotation period. The rotation of the galaxy will switch on a further term in the evolution equations of the magnetic fields: the so-called dynamo term describing the amplification caused in the magnetic flux by the action of a topologically non-trivial bulk velocity field (i.e. $\langle\vec{v} \cdot \vec{\nabla} \times \vec{v}\rangle \neq 0$ ) 113,14. In the presence of the dynamo action the seed field possibly generated by the variation of the internal manifold will be exponentially amplified. The number of e-folds characterizing the amplification is of the order of the number of rotations performed by the galaxy since its origin. In order to allow a successful dynamo action (and a successful genesis of the large scale magnetic field) the original seed field (exponentially amplified later on) should have a specific value. The problem is to check if the seed fields generated from the evolution of the interval dimensions will be strong enough to be amplified to the presently observed value. This analysis will be one of the purposes of the following section.

\section{MAGNETOGENESIS CONSTRAINTS}

The amplified gauge fields should be compared with various constraints coming from general cosmological considerations (critical energy density, possible anisotropies induced in the cosmic microwave background) and from magnetogenesis. If the produced fields are strong enough to seed the galactic dynamo mechanism [13, 14 they should also be compatible with the physics described by the standard model of cosmological evolution.

Suppose that the time evolution of the internal dimensions is monotonic in conformal time. In this case the internal dimensions will either grow or shrink. If the internal dimensions shrink (to Planckian or quasi-Planckian size) they are very small today (of the order of $10^{-33}-10^{-32} \mathrm{~cm}$ ). If they expand they could lead, today, to a smaller (effective) 
four-dimensional Planck mass [33] and to a larger value of $b_{1}$ which could lie between $10^{-33}$ $\mathrm{cm}$ and $10^{-4} \mathrm{~cm}$.

Some theoretical models which could describe the multidimensional evolution are discussed in Section IV. Here a general analysis will be given. The discussion is divided into two parts. In the first part $n$ internal dimensions growing in conformal time will be considered. This cooresponds, within the parametrization of Eq. (2.5), to the case $\lambda<0$. In the second part internal $n$ internal dimensions shrinking in conformal time will be considered. This corresponds to $\lambda>0$ in Eq. (2.5).

\section{A. Internal dimensions growing in conformal time}

According to Eq. (2.5) the growth of the internal dimensions stops in $\eta=-\eta_{1}$ when the radiation dominated phase suddenly begins. The curvature at which the transition to the radiation dominated phase occur is given by $H_{1}=T_{1}^{2} /\left[M_{4}\left(b_{1} M_{4}\right)^{n / 2}\right]$ where $T_{1}$ is the temperature at $-\eta_{1}, M_{4}$ is the four dimensional Planck scale and $H_{1}$ is the Hubble factor $H=\dot{a} / a$ in $-\eta_{1}$. The maximal temperature of the Universe cannot be larger, in this context, than $M_{4}$ so $T_{1} \leq M_{4}$ will be assumed. The present (physical) frequencies relevant for the problem can be obtained using the background evolution during the radiation and matter dominated epochs. After $\eta=-\eta_{1}$, the evolution of the geometry is adiabatic. Thus, the scale corresponding today to $1 / \eta_{1}$ is given by $\omega_{1} \sim 10^{11}\left(M_{4} / M_{P}\right) \mathrm{Hz}$ (where $M_{P} \sim 1.22 \times 10^{19}$ $\mathrm{GeV}$ ). The decoupling frequency (corresponding to the transition from radiation to matter) is $\omega_{\mathrm{dec}} \sim 10^{-16} \mathrm{~Hz}$.

As discussed in the previous Section, thanks to magnetic flux conservation, the ratio between the logarithmic energy spectrum and the radiation energy density is approximately constant 16,17 and, for $T_{1} \sim M_{4}$, it is given by

$$
r(\omega)=\left(\frac{M_{4}}{M_{P}}\right)^{4} \frac{\mathcal{C}(\nu)}{\pi^{2}}\left(\frac{\omega}{\omega_{1}}\right)^{3-2 \nu}
$$

where, for simplicity, we set $\lambda=-\beta$ with $\beta>0$ so that $2 \nu=|n \lambda-1| \equiv n \beta+1$. Notice 
that the effective number of relativistic species at $\eta_{1}$ has been included in the definition of $M_{4}$. Eq. (2.1) can be rewritten as

$$
r(\omega)=\frac{1}{\rho_{\gamma}} \frac{d \rho_{B}}{d \ln \omega}=\left(\frac{M_{4}}{M_{P}}\right)^{2+n \beta} \frac{2^{n \beta-4}}{\pi^{3}}\left(\frac{\omega}{10^{11} \mathrm{~Hz}}\right)^{2-n \beta} \Gamma^{2}\left(\frac{n \beta+1}{2}\right)|n \beta|^{2},
$$

where $\rho_{\gamma}$ is the energy density in radiation. If $n \beta<2$, the logarithmic energy spectrum grows in frequency. The critical energy density constraint is then implemented by requiring that $r\left(\omega_{1}\right)<1$, namely by requiring that the magnetic energy density (evolving according to flux conservation) is smaller than the radiation density. This requirement automatically guarantees, because of the growth in frequency of the logarithmic energy spectrum, that $r(\omega)<1$ also for all $\omega<\omega_{1}$, and in particular, for $\omega_{\mathrm{dec}}$.

If $n \beta>2$ the spectrum decreases in frequency. In order to insure the compatibility with present large scale observations at low frequencies the spectrum should not induce too much anisotropy in cosmic microwave background (CMB). This condition is enforced if $r\left(\omega_{\mathrm{dec}}\right)<10^{-10}$. If $r\left(\omega_{\mathrm{dec}}\right)<10^{-10}$ and $r(\omega)$ decreases in frequency, then, $r(\omega)<1$ for any $\omega_{\text {dec }}<\omega<\omega_{1}$.

Thus, compatibility with the observed features of our present Universe demands

$$
\begin{aligned}
& r\left(\omega_{1}\right)<1, \text { for } n \beta<2, \\
& r\left(\omega_{\mathrm{dec}}\right)<10^{-10}, \text { for } n \beta>2 .
\end{aligned}
$$

Eqs. (2.3) imply, respectively 团,

$$
\begin{aligned}
& 4 \log \left(\frac{M_{4}}{M_{P}}\right)<\log \left[\frac{\pi^{3}}{(n \beta)^{2}}\right]-(n \beta-4) \log 2-2 \log \left[\Gamma\left(\frac{n \beta+1}{2}\right)\right], \quad n \beta<2 \\
& (n \beta+2) \log \left(\frac{M_{4}}{M_{P}}\right)<-8.55+27(2-n \beta)-2 \log n \beta \\
& -2 \log \left[\Gamma\left(\frac{n \beta+1}{2}\right)\right]+(4-n \beta) \log 2, \quad n \beta>2 .
\end{aligned}
$$

Magnetogenesis demands that strong seed fields should be produced. Such a demand 13, 17 translates into the following equations

\footnotetext{
${ }^{4}$ Recall that $\ln$ denotes the Neperian logarithm and log denotes the logarithm in ten basis.
} 


$$
r\left(\omega_{G}\right) \geq 3.7 \times 10^{-33}, \omega_{G} \sim 10^{-14} \mathrm{~Hz}
$$

where $\omega_{G}^{-1} \sim 1$ Mpc. The condition expressed in Eq. (2.6) mildly depends upon the cosmological parameters. Eq. (2.6) assumes $h_{0}=0.65, \Omega_{\mathrm{m}}=0.3$ and $\Omega_{\lambda}=0.7$ and it corresponds to a seed field of $2.5 \times 10^{-16} \mathrm{G}$ at the decoupling epoch. The seed field generated through our mechanism will be exponentially amplified by the galactic rotation. The amplification induced by the differential rotation of the primeval galaxy through the dynamo mechanism goes roughly as $e^{\Gamma t}$ where $\Gamma$ is the dynamo amplification rate and $t$ is the galactic age (roughly 10 Gyrs) [13,14]. In Eq. (2.6) it is assumed that the dynamo amplification rate is of the order of $\Gamma^{-1} \sim 0.5$ Gyr. If the dynamo amplification rate is (more or less artificially) increased [34] the initial seed can be even smaller that the one assumed in Eq. (2.6). For instance if we take $\Gamma^{-1} \sim 0.3$ the seed field is required to satisfy $r\left(\omega_{G}\right)>6.07 \times 10^{-50}$ [34]. According to Eq. (2.6), Eq. (2.2) implies

$$
\begin{aligned}
& (2+n \beta) \log \left(\frac{M_{4}}{M_{P}}\right) \geq \\
& -30.92+25(2-n \beta)-2 \log \left[\Gamma\left(\frac{n \beta+1}{2}\right)\right]-2 \log n \beta-(n \beta-4) \log 2,
\end{aligned}
$$

The constraints imposed by Eqs. (2.4)-(2.5) and by Eq. (2.7) entail a region in the parameter space of the model where the cosmological constraints can be safely satisfied and the magnetogenesis requirements are met. The parameter space (defined by $n \beta$ and by $M_{4} / M_{P}$ ) is illustrated in Fig. 目. The shaded area corresponds to the allowed region. In the shaded area of Fig. 1 (left plot) magnetic fields are produced with growing frequency spectrum. In the shaded area of the right plot of Fig. 1 magnetic fields are produced with decreasing frequency spectrum. The theoretical bias would point, a priori, towards increasing frequency spectra. In fact in this case the two-point functions are decreasing at large distance scales. However, in the present analysis all the possibilities should be borne in mind. The full (thin) line appearing in both plots of Fig. 1 1 denotes a less conservative magnetogenesis requirement and it corresponds to larger a dynamo amplification rate. The thin curve can be obtained by requiring, from Eq. (2.2), $r\left(\omega_{G}\right)>6.07 \times 10^{-50}$ which corresponds to $\Gamma^{-1} \sim 0.5$ Gyr. 


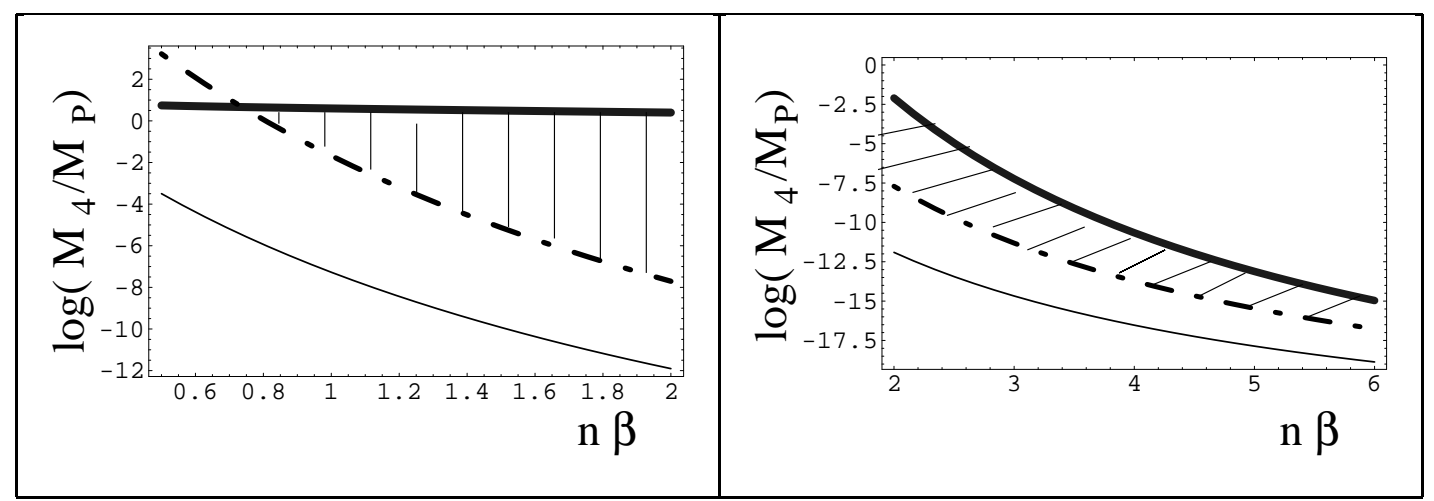

FIG. 1. The allowed region is illustrated for the case of internal dimensions growing in conformal time (i.e. $\beta>0$ ). With the thick (full) line the requirements imposed by cosmological constraints are reported. The constraints set by magnetogenesis are illustrated in the case of a dynamo rate $\Gamma^{-1}=0.5 \mathrm{Gyr}$ (dot-dashed line), and in the (less conservative) case $\Gamma^{-1}=0.3 \mathrm{Gyr}$ (thin line).

According to Fig. 11 reasonable seeds are produced provided $n \beta$ is larger than 0.6 . The four dimensional Planck mass should be small in units of the fundamental Planck mass. The parameter space seems to select typical values of $M_{4}$ between $10^{13} \mathrm{Tev}$ and $10^{3} \mathrm{TeV}$.

These conclusions were reached in the case $T_{1} \simeq M_{4}$. If $T_{1} \ll M_{4}$ similar conclusions can be reached by following the same steps. The conclusion is that the intercepts between the thick and dot-dashed curves are slightly shifted to the left. To give some numerical value, assume, for instance, $T_{1} \sim 10^{-4} M_{4}$. Then we would get that the intercept gets to $n \beta \simeq 0.8$.

In order to avoid confusion we would like to stress that in spite of the fact that, in our case, $M_{4} / M_{P} \ll 1$ the physical size of the internal dimensions is always much smaller than $0.1 \mathrm{~mm}$ corresponding to a $M_{4} \sim \mathrm{TeV}$. Therefore, further constraints coming from submillimiter tests of the Newton law are not directly relevant to our present discussion.

To complete the analysis it is worth mentioning that magnetic fields can be directly constrained from BBN. These bounds are qualitatively different from the ones previously quoted and coming, alternatively, from homogeneity [3] and isotropy [4] of the background geometry at the BBN time. As elaborated in slightly different frameworks through the years 
[35], magnetic fields possibly present at the BBN epoch could have a twofold effect. On one hand they could enhance the rate of reactions (with an effect proportional to $\alpha \rho_{B}$ where $\alpha \sim 1 / 137$ ) and, on the other hand they could artificially increase the expansion rate (with an effect proportional to $\left.\rho_{B}\right)$. It turns out that the latter effect is probably the most relevant. In order to prevent the Universe from expanding too fast at the $\mathrm{BBN}$ epoch $\rho_{B}<0.27 \rho_{\nu}$ where $\rho_{\nu}$ is the energy density contributed by the standard three neutrinos for $T<1 \mathrm{MeV}$. This bound can be imposed also to the spectra discussed in the present Section. Taking into account that the horizon at the BBN epoch (assuming a typical temperature of $0.1 \mathrm{MeV}$ ) corresponds to a present frequency of $\omega_{\mathrm{NS}} \sim 2.28 \times 10^{-12} \mathrm{~Hz}$, the BBN bound implies in the case of the spectra of Eq. (2.2)

$$
\begin{aligned}
& (2+n \beta) \log \left(\frac{M_{4}}{M_{P}}\right)<0.19+(4-n \beta) \log 2+22.6(2-n \beta) \\
& -2 \log \left[\Gamma\left(\frac{n \beta+1}{2}\right)\right]-2 \log n \beta
\end{aligned}
$$

This constraint is qualitatively different from the ones already discussed and that is why it is important to mention it. However, from the quantitative point of view it is roughly equivalent to the critical density constraint. If $n \beta>2$ this bound is always satisfied. If $n \beta<2$ this bounds is a bit more constraining than the critical density limit (by a tiny amount) in the region $1.4<n \beta<2$. Within the numerical accuracy of the present analysis it is not crucial.

Another interesting moment in the life of the Universe where the generated magnetic fields could have an impact is the electroweak epoch [36]. The present frequency corresponding at the electroweak horizon is of the order of $10^{-5} \mathrm{~Hz}$. Fields coherent on that scale can be certainly produced within the present mechanism but they are not constrained by the electroweak physics. 


\section{B. Internal dimensions shrinking in conformal time}

If $\lambda>0$ (internal dimensions shrinking in conformal time), then $2 \nu=|n \lambda-1|$. Therefore, the logarithmic energy spectrum has a twofold form

$$
\begin{aligned}
& r(\omega)=\left(\frac{H_{1}}{M_{P}}\right)^{2}\left(\frac{\omega}{\omega_{1}}\right)^{4-n \lambda} \frac{2^{n \lambda-6}}{\pi^{3}}(2-n \lambda)^{2} \Gamma^{2}\left(\frac{n \lambda-1}{2}\right), \text { for } n \lambda>1 \\
& r(\omega)=\left(\frac{H_{1}}{M_{P}}\right)^{2}\left(\frac{\omega}{\omega_{1}}\right)^{2+n \lambda} \frac{2^{-2-n \lambda}}{\pi^{3}}(n \lambda)^{2} \Gamma^{2}\left(\frac{1-n \lambda}{2}\right), \text { for } n \lambda<1
\end{aligned}
$$

If $n \lambda<4$ the spectrum is always increasing in frequency. On the contrary for $n \lambda>4$ the spectrum decreases and it is peaked in the infra-red. In order to implement the critical density bound for $n \lambda<4, r\left(\omega_{1}\right)<1$ should be demanded. For $n \lambda>4, r\left(\omega_{\mathrm{dec}}\right)<10^{-10}$

should be required in order to guarantee the compatibility of our toy model with the CMB anisotropies. Then, using Eq. (2.9), the critical density bound demands

$$
\begin{aligned}
& 2 \log \left(\frac{H_{1}}{M_{P}}\right) \leq(2+n \lambda) \log 2+\log \pi^{3}-2 \log n \lambda-2 \log \left[\Gamma\left(\frac{1-n \lambda}{2}\right)\right], \quad n \lambda<1 . \\
& 2 \log \left(\frac{H_{1}}{M_{P}}\right) \leq(6-n \lambda)+\log \pi^{3} \\
& -2 \log (n \lambda-2)-2 \log \left[\Gamma\left(\frac{n \lambda-1}{2}\right)\right], \quad 1<n \lambda<4 .
\end{aligned}
$$

In this case the constraints are expressed using curvature (rather than temperature ) scales. This is because $H_{1} \sim 1 /\left(a_{1} \eta_{1}\right)$ cannot be much smaller than $M_{P}$ In this language the (present) maximal frequency turns out to be $\omega_{1}=10^{11} \sqrt{H_{1} / M_{P}}$ Hz. Using Eq. (2.9) in the case $n \lambda>4$ the CMB bound becomes

$$
\begin{aligned}
& n \lambda \log \left(\frac{H_{1}}{M_{P}}\right) \leq \\
& -17.01+54(4-n \lambda)-2(n \lambda-6) \log 2-2 \log (2-n \lambda)^{2}-4 \log \left[\Gamma\left(\frac{n \lambda-1}{2}\right)\right]
\end{aligned}
$$

Finally the magnetogenesis requirements [already introduced in Eq. (2.6)] impose

$$
\begin{aligned}
& (2-n \lambda) \log \left(\frac{H_{1}}{M_{P}}\right) \geq-61.88+50(2+n \lambda) \\
& +2(2+n \lambda) \log 2-4 \log n \lambda-4 \log \left[\Gamma\left(\frac{1-n \lambda}{2}\right)\right], \quad n \lambda<1
\end{aligned}
$$




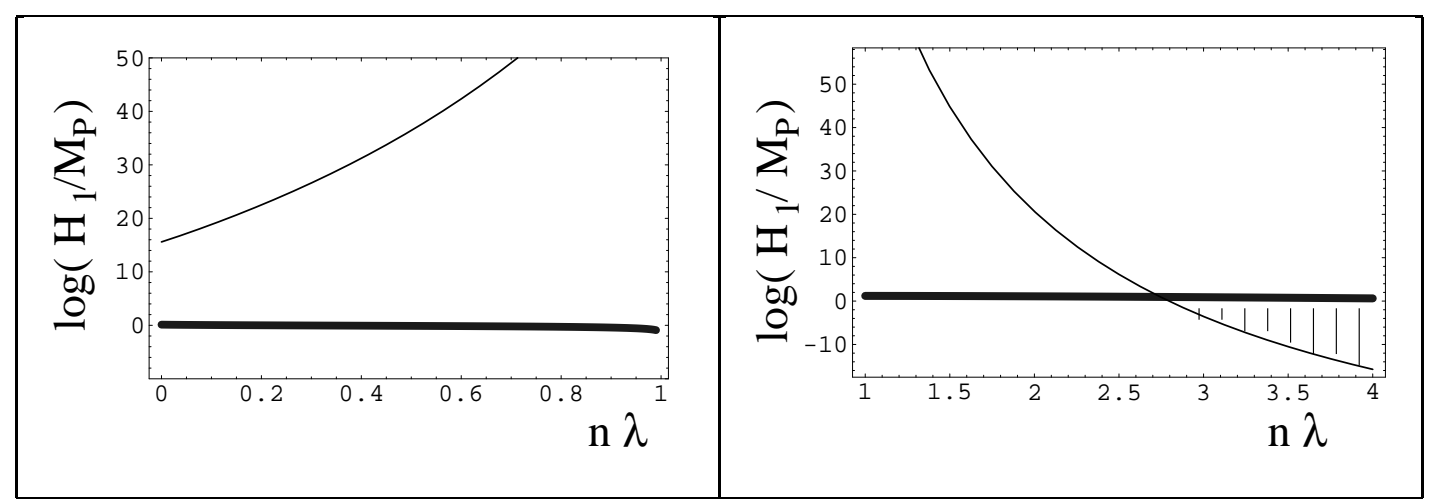

FIG. 2. The allowed region in the case of $\lambda>0$ is illustrated. The magnetogenesis requirements following from Eqs. (2.14) are reported with full (thin) lines. The thick lines denote the cosmological bounds. For $n \lambda<1$ magnetogenesis cannot take place since there is no intercept between the cosmological bounds and the magnetogenesis region. If $n \lambda>1$ magnetogeneis demands $n \lambda>2.7$.

$$
\begin{aligned}
& n \lambda \log \left(\frac{H_{1}}{M_{P}}\right) \geq-61.88+50(4-n \lambda) \\
& -2(n \lambda-6) \log 2-4 \log (n \lambda-2)-4 \log \left[\Gamma\left(\frac{n \lambda-1}{2}\right)\right], \quad n \lambda>1
\end{aligned}
$$

Fig. 2 shows that, once the cosmological bounds are imposed, magnetogenesis is possible only of the parameter space implying that $n \lambda>1$. If $n \lambda<1$ magnetogenesis is excluded unless the produced magnetic fields over-close the Universe. This possibility must be rejected.

\section{TAILORING THE MODEL OF INTERNAL EVOLUTION}

In the previous Sections it has been established that large scale magnetic fields can be produced either if $n \beta \geq 0.6$ (if the internal dimensions grow in conformal time) or if $n \lambda>2.7$ (if the internal dimensions shrink in conformal time). Some toy model of internal evolution will now be analyzed. The purpose of this analysis is to show that the physical requirements coming from magnetogenesis and from other cosmological considerations can be indeed realized in specific models of dynamical evolution of a $D$-dimensional background geometry. 


\section{A. General considerations}

Consider the $D$-dimensional action for a scalar field, minimally coupled to gravity:

$$
S=S_{g}+S_{m}=-\int d^{D} x \sqrt{-g} R+\int d^{D} x \sqrt{-g}\left[\frac{1}{2} g^{\alpha \beta} \partial_{\alpha} \varphi \partial_{\beta} \varphi-V(\varphi)\right]
$$

where we work, for simplicity, in Planck units. We shall consider a homogeneous, Bianchitype I metric background, whose spatial part is the product of two conformally flat manifolds as introduced in Eq. (2.1). For the background given in Eq. (2.1), the equations of motion obtained by varying the action with respect to $g_{\mu \nu}$ and $\varphi$,

$$
R_{\mu}^{\nu}-\frac{1}{2} \delta_{\mu}^{\nu} R=\frac{1}{2}\left[\partial_{\mu} \varphi \partial^{\nu} \varphi-\frac{1}{2} \delta_{\mu}^{\nu} g^{\alpha \beta} \partial_{\alpha} \varphi \partial_{\beta} \varphi+\delta_{\mu}^{\nu} V(\varphi)\right], \quad g^{\alpha \beta} \nabla_{\alpha} \nabla_{\beta} \varphi+\frac{\partial V}{\partial \varphi}=0
$$

reduce simply to

$$
\begin{gathered}
d(d-1) \mathcal{H}^{2}+n(n-1) \mathcal{F}^{2}+2 n d \mathcal{H} \mathcal{F}=\left(\frac{\varphi^{\prime 2}}{2}+a^{2} V\right), \\
2(d-1) \mathcal{H}^{\prime}+(d-1)(d-2) \mathcal{H}^{2}+2 n \mathcal{F}^{\prime}+n(n+1) \mathcal{F}^{2}+2 n(d-2) \mathcal{H} \mathcal{F}=\left(a^{2} V-\frac{\varphi^{\prime 2}}{2}\right), \\
2(n-1) \mathcal{F}^{\prime}+2 d \mathcal{H}^{\prime}+d(d-1) \mathcal{H}^{2}+n(n-1) \mathcal{F}^{2}+2(d-1)(n-1) \mathcal{H} \mathcal{F}=\left(a^{2} V-\frac{\varphi^{\prime 2}}{2}\right), \\
\varphi^{\prime \prime}+[(d-1) \mathcal{H}+n \mathcal{F}] \varphi^{\prime}+\frac{\partial V}{\partial \varphi}=0,
\end{gathered}
$$

where $\mathcal{H}=(\ln a)^{\prime}, \mathcal{F}=(\ln b)^{\prime}$.

These equations are not all independent, and the scalar field equation, for instance, can be obtained from the other Einstein equations. By summing and subtracting the above equations one obtains

$$
\begin{aligned}
a^{2} V & =[(d-1) \mathcal{H}+n \mathcal{F}]^{\prime}+[(d-1) \mathcal{H}+n \mathcal{F}]^{2}, \\
\frac{1}{2} \varphi^{\prime 2} & =-[(d-1) \mathcal{H}+n \mathcal{F}]^{\prime}+(d-1) \mathcal{H}^{2}-n \mathcal{F}^{2}+2 n \mathcal{H} \mathcal{F}, \\
\mathcal{F}^{\prime}-\mathcal{H}^{\prime} & =-(\mathcal{F}-\mathcal{H})[(d-1) \mathcal{H}+n \mathcal{F}] .
\end{aligned}
$$

Consider now the case where the external and the internal scale factors can be parametrized as $a(\eta) \sim(-\eta)^{\sigma}$ and $b(\eta) \sim(-\eta)^{\lambda}$. If $V=0$ and $\varphi=0$ we have that the well known solution 
of this sysmtem is indeed given by a power law behaviour of the scale factors provided the exponents satisfy, according to Eqs. (3.4)-(3.6)

$$
\begin{aligned}
& (d-1) \sigma+n \lambda=1, \\
& (d-1) \sigma^{2}+n \lambda^{2}=1+2 \lambda .
\end{aligned}
$$

These two conditions can be also easily expressed in cosmic time . In this case we have to recall that $a(\eta) d \eta=d t$. Therefore if we parameterize the solutions in cosmic time as

$$
a(t) \sim(-t)^{\epsilon}, \quad b(t) \sim(-t)^{\zeta}
$$

we will have that the exponents in cosmic and conformal time are simply related as $\epsilon=$ $\sigma /(\sigma+1)$ and $\zeta=\lambda /(\sigma+1)$. Using these two last relations into Eqs. (3.7)-(3.8) the two standard Kasner conditions are recovered, namely $d \epsilon+n \zeta=1$ and $d \epsilon^{2}+n \zeta^{2}=1$. The solution of Eqs. (3.7)-(3.8) gives, for each dimension $n$ of the internal space, two conjugate solutions which we write in the case $d=3$ :

$$
\begin{aligned}
& \sigma_{ \pm}=\mp \frac{1}{2}\left[\sqrt{\frac{3 n}{n+2}} \mp 1\right], \\
& \lambda_{ \pm}= \pm \sqrt{\frac{3}{n(n+2)}} .
\end{aligned}
$$

The related cosmic time exponents can be easily obtained

$$
\begin{gathered}
\epsilon_{ \pm}=\frac{1 \mp \sqrt{\frac{n+2}{3 n}}}{1 \mp 3 \sqrt{\frac{n+2}{3 n}}}, \\
\zeta_{ \pm}=\frac{-2}{n\left(1 \mp 3 \sqrt{\frac{n+2}{3 n}}\right)} .
\end{gathered}
$$

In the parameterization of Eq. (3.9) the solution labeled by + in the exponents of the internal manifold denote a growing solution whereas the solution denoted by - denote a shrinking solution. In order to produce large scale magnetic fields we should require that, when the internal dimensions expand,

$$
\left|n \lambda_{-}\right|>0.6
$$


If we now insert the explicit expression for $\lambda_{-}$we have that Eq. (3.12) implies

$$
\sqrt{\frac{3 n}{n+2}} \geq 0.6
$$

which is satisfied for $n=1$ and it always satisfied for any $n>1$. Since $\lambda_{-}$is negative for any $n b(\eta) \sim(-\eta)^{\lambda_{-}}$will be growing for any $n$. It is useful to look also at the cosmic time picture. In cosmic time $b(t) \sim(-t)^{\zeta_{-}}$. Since $\dot{b}>0$ for any $n$ the solution is expanding also in cosmic time 包.

In order to produce large scale magnetic fields when the internal dimensions shrink according to the Kasner solutions we have to require

$$
n \lambda_{+}>2.7
$$

If we insert the explicit expression of $\lambda_{+}$we can see that Eq. (3.14) imposes the following inequality

$$
\sqrt{\frac{3 n}{n+2}} \geq 2.7
$$

which can never be satisfied for any $n$. If the dynamics of the internal dimensions follows vacuum (Kasner) solutions, then, the requirement of generating large scale magnetic fields compatible with the cosmological bounds automatically selects an expanding dynamics of the internal dimensions.

Vacuum Kasner solutions can be generalized to the case of vanishing scalar potential. In this case the equations of motion can be always solved and the power-law ansatz can still be made for the scale factors. The solutions will be given by:

$$
\begin{aligned}
& (d-1) \sigma+n \lambda=1 \\
& \varphi^{\prime 2}=2\left\{1-(d-1) \sigma^{2}-n \lambda^{2}+2 \sigma\right\} \frac{1}{\eta^{2}}
\end{aligned}
$$

\footnotetext{
${ }^{5}$ Notice that the the over-dot denotes derivation with respect to cosmic time. The kinematical conditions on the expansions or contraction of a given background geometry should be stated in cosmic rather than in conformal time.
} 
Also in this case we can easily show that if the solution is contracting (in the internal manifold) large scale magnetic fields cannot be produced. Indeed if $b(\eta)$ contracts we will have that the allowed range of $n \lambda$ is given by $0<n \lambda<d$. If we take $d=3$ we see that $n \lambda<3$. But according to our analysis $n \lambda>2.7$. Therefore only a tiny slice of parameter space (i.e. $2.7<n \lambda<3$ ) could give a positive result.

\section{B. Explicit examples}

Consider, as an example, the case $n=6$. In this case the internal manifold has six internal scale factors $b(\eta)$. As assumed in Eq. (2.1) both the internal and the external metrics will be maximally symmetric. Suppose that the internal dimensions are then expanding according to the Kasner solutions, namely $b(\eta) \sim(-\eta)^{\lambda_{-}}$and $a(\eta) \sim(-\eta)^{\sigma_{-}}$. If $n=6 \lambda_{-}=-1 / 4$ and $\sigma_{-}=5 / 4$. The the background model given in Eq. (2.5) will be in this case

$$
\begin{array}{ll}
a(\eta)=a_{1}\left(-\frac{\eta}{\eta_{1}}\right)^{5 / 4}, \quad b(\eta)=b_{1}\left(-\frac{\eta}{\eta_{1}}\right)^{-1 / 4}, & \eta<-\eta_{1}, \\
a(\eta)=a_{1}\left(\frac{\eta+2 \eta_{1}}{\eta_{1}}\right), \quad b(\eta)=b_{1}, & -\eta_{1} \leq \eta \leq \eta_{2},
\end{array}
$$

where a sudden match to the radiation dominated epoch is assumed in $-\eta_{1}$. The internal scale factors grow from $\eta \rightarrow-\infty$ up to $-\eta_{1}$. The external scale factor contracts. This is because of the mathematical nature of vacuum Kasner solutions. Using Eqs. (2.3) and (2.6) -(2.11) the evolution of the mode functions during the multidimensional phase will be given by

$$
\phi_{k}^{\prime \prime}-\left[k^{2}-\frac{21}{16 \eta^{2}}\right] \phi_{k}=0,
$$

whose solution is given by Eq. (2.12) with $\nu=5 / 4$. At the time $-\eta_{1}$ the background will pass to radiation with a temperature $T_{1} \sim M_{4}$. The produced large scale magnetic fields will evolve according to flux conservation and the ratio between the magnetic and radiation energy density is roughly constant. Then, from Eqs. (2.19)-(2.1), $r(\omega)$ can be explicitly written as 


$$
r(\omega)=\left(\frac{M_{4}}{M_{P}}\right)^{4} \frac{\mathcal{C}(5 / 4)}{\pi^{2}}\left(\frac{\omega}{\omega_{1}}\right)^{1 / 2}, \omega_{\mathrm{dec}}<\omega<\omega_{1}
$$

where $\omega_{1} \sim 10^{11}\left(M_{4} / M_{P}\right) \mathrm{Hz}$ and $\omega_{\mathrm{dec}} \sim 10^{-16} \mathrm{~Hz}$ are both evaluated at the present time and where $\mathcal{C}(5 / 4) / \pi^{2} \sim 1.86 \times 10^{-3}$ [see Eq. (2.17)]. The value of $r(\omega)$ at the scale relevant for magnetogenesis (i.e. $\omega_{G} \sim 10^{-14} \mathrm{~Hz}$ ) is given by

$$
r\left(\omega_{G}\right)=1.86 \times 10^{-15.5}\left(\frac{M_{4}}{M_{P}}\right)^{3.5} .
$$

As discussed in Eq. (2.6), in order to have a successful magnetogenesis $r\left(\omega_{G}\right)>10^{-33}$ should be required. Imposing this requirement it turns out that from Eq. 3.20

$$
\left(\frac{M_{4}}{M_{P}}\right) \geq 10^{-5}
$$

If a less conservative magnetogenesis requirement is assumed (i.e. $r\left(\omega_{G}\right) \geq 10^{-50}$ ) it can be checked that

$$
\left(\frac{M_{4}}{M_{P}}\right) \geq 10^{-10}
$$

should be demanded. If $M_{4} \sim 10^{12} \mathrm{TeV}$ both Eqs. (3.21)-(3.22) are satisfied and $\omega_{1} \sim 10^{7}$ Hz. Moreover, $r\left(\omega_{\mathrm{dec}}\right) \sim 10^{-30}$ with negligible impact on the CMB. If $M_{4} \sim 10^{7} \mathrm{TeV}$ large scale magnetic fields can be generated only if a higher dynamo amplification rate is assumed, so that Eq. (3.22) can be applied. In this case $\omega_{1} \sim 100 \mathrm{~Hz}$.

\section{DISCUSSION AND CONCLUSIONS}

In this paper the possible generation of large scale magnetic fields in the case of dynamical internal dimensions has been considered. The time dependence of internal dimensions (a generic ingredient of Kaluza-Klein models and of superstring theories) is severely constrained by our knowledge of BBN. The fact that internal dimensions could not be dynamical after the Universe was old of approximately one second does not exclude that they could have been dynamical for temperatures larger that $1 \mathrm{MeV}$. 
Since internal dimensions naturally break the conformal invariance of the evolution equations of Abelian gauge fields propagating in the four-dimensional world, electromagnetic fluctuations can be amplified. The typical amplitude and scale of the produced magnetic fields has been investigated under the assumption that the internal dimensions were dynamically evolving prior to the radiation dominated epoch.

In this context the problem of generating a seed field for the galactic dynamo action has been studied. The problem of magnetogenesis amounts to satisfying the numerical requirements necessary for a successful dynamo action together with all the bounds imposed by the standard model of cosmological evolution. If the conformal time evolution of the internal dimensions is monotonic and if the multidimensional phase is suddenly matched to radiation, then magnetogenesis is possible provided the dimensions belonging to the internal manifold are expanding. If the internal dimensions are contracting magnetogenesis is not possible.

The considerations reported in this paper should emerge as a part of a complete cosmological scenario. This problem has been only partially addressed in our considerations and further work is certainly needed. However, the idea of connecting large scale magnetic fields with the dynamics of internal dimensions seems to be of some interest.

\section{ACKNOWLEDGMENTS}

The author wishes to thank M. E. Shaposhnikov for useful discussions and valuable exchanges of ideas. 


\section{REFERENCES}

[1] D. N. Schramm, R. V. Wagoner, Ann. Rev. Nucl. Part. Sci. 27, 37 (1977) ;S. Sarkar, Rept. Prog. Phys. 59, 1493 (1996).

[2] V. A. Rubakov and M. E. Shaposhnikov Usp.Fiz.Nauk 166,493 (1996) [Phys.Usp. 39, 461 (1996)].

[3] K. Jedamzik and G. M. Fuller, Astrophys. J. 423, 33 (1994); H. Kurki-Suonio K. Jedamzik and G.J. Mathews, Astrophys. J. 479, 31 (1997); K. Kainulainen, H. KurkiSuonio, E. Sihvola, Phys.Rev.D 59083505 (1999).

[4] K. S. Thorne, Astrophys. J. 148, 51 (1967); S. W. Hawking and R. J. Tayler, Nature 309, 1278 (1966); J. D. Barrow, Mon. Not. R. Astron. Soc. 175, 359 (1976).

[5] G. Steigman, Ann. Rev. Astron. Astroph. 14, 339 (1976).

[6] J. B. Rehm and K. Jedamzik, Phys.Rev.Lett. 81, 3307 (1998); H. Kurki-Suonio and E. Sihvola, Phys. Rev. Lett. 843756 (2000); J. B. Rehm and K. Jedamzik, astroph/0006381; H. Kurki-Suonio and E. Sihvola, astro-ph/0006448.

[7] J. D. Barrow, Phys. Rev. D 35, 1805 (1987).

[8] E. W. Kolb, M. J. Perry, and T.P. Walker, Phys. Rev. D 33, 869 (1986); F. S. Accetta, L. M. Krauss, and P. Romanelli, Phys. Lett. B 248, 146 (1990).

[9] T. Appelquist, A. Chodos, and P. G. O. Freund, Modern Kaluza Klein Theories (Addison-Wesley, Redwood City, CA, 1987).

[10] M. B. Green, J. H. Schwartz, and E. Witten, Superstring Theory (Cambridge University Press, Cambridge, England, 1987).

[11] E. Fermi, Phys. Rev. 75. 1169 (1949); J. Schwinger, Phys. Rev. 75, 1912 (1949).

[12] E. R. Harrison, Phys. Rev. Lett. 30, 188 (1973). 
[13] Y. B. Zeldovich, A. A. Rusmaikin, and D. D. Sokoloff, Magnetic Fields in astrophysics (Gordon and Breach, New York, 1983).

[14] P. P. Kronberg, Rep. Prog. Phys. 57, 325 (1994).

[15] R. M. Kulsrud, R. Cen, J. P. Ostriker and D. Ryu, Astrophys. J. 480, 481 (1997); R. M. Kulsrud, Ann. Rev. Astron. Astroph. 37, 37 (1999).

[16] M. S. Turner and L. M. Widrow, Phys. Rev. D 37, 2734 (1988);T. Vachaspati and A. Vilenkin, Phys.Rev.Lett. 67, 1057 (1991); B. Ratra, Astrophys. J. Lett. 391, L1 (1992); S. Carroll, G. Field and R. Jackiw, Phys. Rev. D 41, 1231 (1990); W. D. Garretson, G. Field and S. Carroll, Phys. Rev. D 46, 5346 (1992); G. B. Field and S. M. Carroll, astro-ph/9811206; A. Dolgov and J. Silk, Phys. Rev. D 47, 3144 (1993); A. Dolgov, Phys.Rev.D 48, 2499 (1993).

[17] M. Gasperini, M. Giovannini and G. Veneziano, Phys. Rev. Lett. 75 (1995) 3796; M. Gasperini, M. Giovannini, and G. Veneziano Phys. Rev. D 52, 6651 (1995); M. Giovannini Phys. Rev. D 58124027 (1998).

[18] T. Vachaspati, Phys. Lett. B 265, 258 (1991); K. Enqvist and P. Olesen, ibid., 319, 178 (1993); ibid. 329, 195 (1994); T. W. Kibble and A. Vilenkin, Phys. Rev. D 52, 679 (1995); G. Baym, D. Bodeker and L. McLerran, ibid. 53, 662 (1996); G. Sigl, A. V. Olinto and K. Jedamzik, Phys. Rev. D 55, 4852 (1997); M. Joyce and M. E. Shaposhnikov, Phys. Rev. Lett. 791193 (1997).

[19] M. Kamionkowski and Arthur Kosowsky, Ann.Rev.Nucl.Part.Sci. 49, 77 (1999); A. Kosowsky and A. Loeb, Astrophys. J. 461, 1 (1996); D. Harari, J. Hayaward, and M. Zaldarriaga, Phys. Rev. D 55, 1841 (1997); M. Giovannini, Phys. Rev. D 56, 3198 (1997); J. Adams, U. H. Danielsson, D. Grasso, and H. Rubinstein, Phys. Lett. B 388 253 (1996); R. Durrer, T. Kahniashvili, and A. Yates Phys. Rev. D 58, 123004 (1998); J. Barrow and K. Subramanian, Phys. Rev. Lett. 81, 3575 (1998); R. Durrer, P.G. 
Ferreira, T. Kahniashvili, Phys. Rev. D 61, 043001 (2000); K. Jedamzik, V. Katalinic and A. Olinto, astro-ph/991110.

[20] L. P. Grishchuk, Sov. Phys. JETP 40, 409 (1975); Sov. Phys. Usp. 31, 940 (1988).

[21] L. Parker, Phys. Rev. Lett. 21, 562 (1968); Phys. Rev. 183, 1057 (1969).

[22] P. G. Bergmann, Int. J. Theor. Phys. 1, 25 (1968).

[23] M. Giovannini, Phys. Rev. D 55, 595 (1997).

[24] L. H. Ford, Phys. Rev. D 31, 704 (1985).

[25] M. Abramowitz and I. A. Stegun, Handbook of mathematical functions (Dover, New York, 1972).

[26] N. D. Birrel and P. C. W. Davies, Quantum fields in curved space (Cambridge University Press, Cambridge 1982).

[27] J. Garriga and E. Verdaguer, Phys. Rev. D 39, 1072 (1991).

[28] M. Gasperini and M. Giovannini, Phys. Lett. B 282, 36 (1992); Phys.Rev.D 47, 1519 (1993).

[29] M. Giovannini and M. E. Shaposhnikov, hep-ph/0004269.

[30] D. Biskamp, Non-Linear Magnetohydrodynamics (Cambridge University Press, Cambridge, England, 1994); N. A. Krall and A. W. Trivelpiece, Principles of Plasma Physics (San Francisco Press, San Francisco 1986).

[31] M. Giovannini, Phys.Rev.D 61, 063502 (2000).

[32] A. Brandenburg, K. Enqvist, and P. Olesen, Phys. Lett. B 392, 395 (1997); Phys. Rev. D 54, 1291 (1996); P. Olesen, Phys. Lett. B 398, 321 (1997).

[33] I. Antoniadis, S. Dimopoulos, G. Dvali Nucl.Phys.B 51670 (1998); N. Arkani-Hamed, S. Dimopoulos, G. Dvali, Phys.Lett.B 429263 (1998); I. Antoniadis, N. Arkani-Hamed, 
S. Dimopoulos, G. Dvali, Phys.Lett.B 436257 (1998); N. Arkani-Hamed, S. Dimopoulos, N. Kaloper, J. March-Russell, Nucl.Phys.B567, 189 (2000); N. Arkani-Hamed, S. Dimopoulos, J. March-Russel, hep-th/9809124.

[34] A. A. Rusmaikin, D. D. Sokolov, and V. I. Turchainov, Astron. Zh. 57, 311 (1980) [Sov. Astron. 24, 182 (1980)]; A. C. Davis, M. Lilley, and O. Tornkvist, Phys. Rev. D 60, 021301 (1999).

[35] G. Greenstein, Nature 223, 938 (1969); J. J. Matese and R. F. O' Connel, Astrophys. J. 160, 451 (1970); B. Cheng, D. N. Schramm and J. Truran, Phys. Rev. D 45, 5006 (1994); B. Cheng, A. Olinto, D. N. Schramm and J. Truran, Phys. Rev. D 54, 4174 (1996); D. Grasso and H. Rubinstein, Astropart. Phys. 3, 95 (1995) and Phys. Lett. B 379, 73 (1996); P. Kernan, G. Starkman and T. Vachaspati, Phys. Rev. D 54, 7207 (1996).

[36] M. Giovannini and M. E. Shaposhnikov, Phys. Rev. D 57, 2186 (1998); Phys. Rev. Lett. 80, 22 (1998); K. Kajantie, M. Laine, J. Peisa, K. Rummukainen, M. E. Shaposhnikov, Nucl.Phys.B 544, 357 (1999); P. Elmfors, K. Enqvist, and K. Kainulainen, Phys.Lett.B 440 269, (1998); M. Giovannini, Phys.Rev.D 61, 063004 (2000); M. Laine, hep-ph/0001292. 